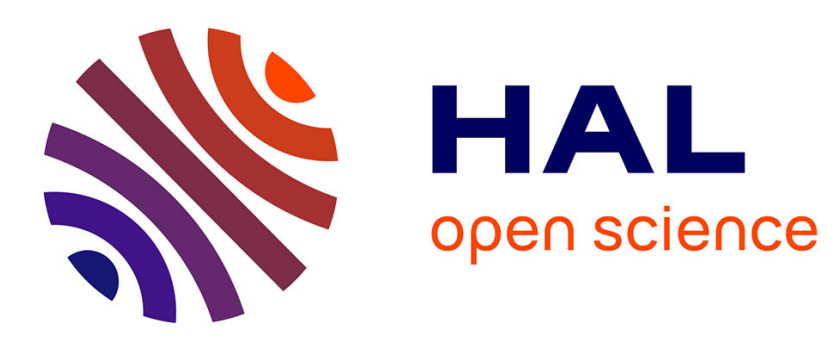

\title{
Spectromètre à haute résolution utilisant un laser à fréquence accordée par effet Zeeman
}

\author{
Henri Brunet
}

\section{To cite this version:}

Henri Brunet. Spectromètre à haute résolution utilisant un laser à fréquence accordée par effet Zeeman. Revue de Physique Appliquée, 1967, 2 (3), pp.191-197. 10.1051/rphysap:0196700203019100 . jpa00242790

\section{HAL Id: jpa-00242790 https://hal.science/jpa-00242790}

Submitted on 1 Jan 1967

HAL is a multi-disciplinary open access archive for the deposit and dissemination of scientific research documents, whether they are published or not. The documents may come from teaching and research institutions in France or abroad, or from public or private research centers.
L'archive ouverte pluridisciplinaire HAL, est destinée au dépôt et à la diffusion de documents scientifiques de niveau recherche, publiés ou non, émanant des établissements d'enseignement et de recherche français ou étrangers, des laboratoires publics ou privés. 


\title{
SPEGTROMÈTRE A HAUTE RÉSOLUTION UTILISANT UN LASER A FRÉQUENGE AGGORDÉE PAR EFFET ZEEMAN

\author{
Par HENRI BRUNET, \\ Gentre de Recherches de la Compagnie Générale d'Électricité, \\ Département Recherches Physiques de Base, route de Nozay, Marcoussis (Essonne).
}

\begin{abstract}
Résumé. - Nous avons étudié le déplacement de fréquence, par effet Zeeman, de raies laser infrarouges du néon et du xénon, de longueurs d'onde proches de 7,5 $\mu$.

Les déplacements de fréquence obtenus, de l'ordre de $0,1 \mathrm{~cm}^{-1}$, sont suffisamment élevés pour que l'on puisse réaliser un spectromètre infrarouge de très haute résolution permettant d'analyser les spectres de rotation-vibration de molécules sur un intervalle de $\pm 0,1 \mathrm{~cm}^{-1}$.

Quelques bandes d'absorption de molécules polyatomiques, $\mathrm{CH}_{4}, \mathrm{C}_{2} \mathrm{H}_{6}, \mathrm{SF}_{6}$ et $\mathrm{C}_{3} \mathrm{H}_{8}$, ont été étudiées entre 1200 et $1400 \mathrm{~cm}^{-1}$ avec une résolution meilleure que $0,005 \mathrm{~cm}^{-1}$.

Abstract. - The frequency shifts of infrared laser lines, obtained by the Zeeman effect, have been studied for wavelengths around $7.5 \mu$.

The observed frequency shifts, up to $0.1 \mathrm{~cm}^{-1}$, are large enough to realize a tuned-infrared laser spectrometer having a resolving power of more than 1 in $3 \times 10^{5}$ and the rotation-vibration spectra of molecules can be studied in a range of $\pm 0.1 \mathrm{~cm}^{-1}$ around the laser frequency.

1 Several absorption bands of the polyatomic molecules $\mathrm{CH}_{4}, \mathrm{C}_{2} \mathrm{H}_{6}, \mathrm{C}_{3} \mathrm{H}_{8}$ and $\mathrm{SF}_{6}$ have been studied in the region 1250 to $1400 \mathrm{~cm}^{-1}$ using neon and xenon laser lines, the resolution being better than $0.005 \mathrm{~cm}^{-1}$.
\end{abstract}

Introduction. - Plusieurs transitions laser infrarouges du néon et du xénon, de longueurs d'onde proches de $7,5 \mu$, ont des gains suffisamment élevés pour que leurs fréquences puissent être déplacées de $\pm 0,1 \mathrm{~cm}^{-1}$ par effet Zeeman.

On dispose alors d'une source infrarouge d'une largeur spectrale très étroite, de fréquence presque continûment variable sur une plage de $\pm 0,1 \mathrm{~cm}^{-1}$, ce qui permet d'analyser, avec une résolution de l'ordre de 500000 à $1400 \mathrm{~cm}^{-1}$, les spectres de rotation-vibration des molécules qui absorbent l'émission laser.

Gette méthode a été utilisée, avec succès, par plusieurs chercheurs, pour l'étude à très haute résolution de spectres de molécules polyatomiques au voisinage de $3,39 \mu$ [1], $3,5080 \mu$ [2] et 7,5 $\mu$ [3].

Après avoir exposé le principe de la spectroscopie par effet Zeeman, nous donnons les résultats originaux obtenus sur des bandes d'absorption de $\mathrm{CH}_{4}, \mathrm{C}_{2} \mathrm{H}_{6}$, $\mathrm{SF}_{6}$ et $\mathrm{C}_{3} \mathrm{H}_{8}$ grâce aux raies laser du néon à 7,4799 $\mu$, $7,6510 \mu, 7,7015 \mu, 7,7655 \mu, 8,0008 \mu$ et à la raie du xénon à $7,3167 \mu$.

I. Fonctionnement du laser à fréquence accordée. I.1. EFFET DU CHAMP MAGNÉTIQUE SUR LE GOEFFICIENT D'AMPLIFICATION. - En présence d'un champ magné-

(1) Cette étude a été subventionnée par la Direction des Recherches et Moyens d'Essais. tique suffisamment élevé, la courbe de gain initialement centrée en $\nu_{0}$ se décompose en trois groupes de courbes : les composantes $\sigma_{i}^{+}$centrées en $v_{0}+\Delta v_{i}(H)$, les composantes $\sigma_{i}^{-}$centrées en $\nu_{0}-\Delta v_{i}(H)$ et les composantes $\pi_{i}$ centrées en $v_{0}+\delta v_{i}$.

Lorsque le champ magnétique est appliqué parallèlement à l'axe du laser, on observe exclusivement les composantes $\sigma_{i}^{+}$et $\sigma_{i}^{-}$, polarisées circulairement, à droite et à gauche. Par contre, lorsque le champ magnétique est transversal, on observe les composantes $\sigma_{i}^{+}, \sigma_{i}^{-}$et $\pi_{i}$, avec des polarisations linéaires, les composantes $\sigma$ ayant une direction de polarisation perpendiculaire à celles des $\pi$.

Les déplacements de fréquence dus à l'effet Zeeman sont :

$$
\begin{aligned}
\Delta v_{i}(H) & =\mu_{\mathrm{B}} H\left(g_{2} m_{2}-g_{1} m_{1}\right)=\mu_{\mathrm{B}} g_{\text {eff }} H \\
\delta v_{i}(H) & =\mu_{\mathrm{B}} H m_{2}\left(g_{2}-g_{1}\right)
\end{aligned}
$$

$g_{1}$ et $g_{2}$ sont les facteurs de Landé des niveaux supérieur et inférieur,

$m_{1}$ et $m_{2}$ les nombres quantiques magnétiques, $\mu_{B}$ le magnéton de Bohr.

Les déplacements notés $\Delta v_{i}$ sont obtenus en associant à chaque valeur de $m_{1}$ les valeurs correspondantes de $m_{2}, m_{2}=m_{1}, m_{2}=m_{1} \pm 1$.

Les courbes de gain, en champ magnétique longitudinal, sont de la forme [4] :

$$
g_{ \pm}^{i}=g_{0}^{i} \mathrm{e}^{-\left(\nu^{\prime} \pm \Delta v_{i}^{\prime}\right)^{2}}
$$


TABLEAU I

\begin{tabular}{|c|c|c|c|c|c|}
\hline$\lambda(\mu)-\nu\left(\mathrm{cm}^{-1}\right)$ & $\begin{array}{l}\text { TRANSITION } \\
\qquad \begin{array}{l}m \rightarrow m^{\prime} \\
-\end{array}\end{array}$ & $\underline{g_{\text {eff }}}$ & $\begin{array}{l}\text { INTENSITÉS } \\
\text { RELATIVES } \\
-\end{array}$ & $\begin{array}{c}\Delta v_{i} \\
\mathrm{Mc} / \mathrm{s} / \propto\end{array}$ & $\begin{array}{c}\Delta \nu \text { MESURÉ } \\
\mathrm{Mc} / \mathrm{s} / \propto \\
-\end{array}$ \\
\hline $\begin{array}{c}7,7015-(1298,443) \\
\text { Néon } 4 p^{\prime}[3 / 2]_{2}-3 d^{\prime}[5 / 2]_{3}\end{array}$ & 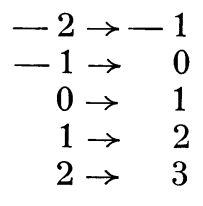 & $\begin{array}{l}1,243 \\
1,184 \\
1,125 \\
1,066 \\
1,007\end{array}$ & $\begin{array}{r}1 / 35 \\
3 / 35 \\
6 / 35 \\
10 / 35 \\
15 / 35\end{array}$ & $\begin{array}{l}1,74 \\
1,66 \\
1,57 \\
1,49 \\
1,41\end{array}$ & 1,53 \\
\hline $\begin{array}{c}7,7655-(1287,755) \\
\text { Néon } 4 p^{\prime}[1 / 2]_{1}-3 d^{\prime}[3 / 2]_{2}\end{array}$ & $\begin{aligned}-1 & \rightarrow 0 \\
0 \rightarrow & 1 \\
1 \rightarrow & 2\end{aligned}$ & $\begin{array}{l}1,397 \\
1,242 \\
1,087\end{array}$ & $\begin{array}{l}1 / 10 \\
3 / 10 \\
6 / 10\end{array}$ & $\begin{array}{l}1,96 \\
1,74 \\
1,52\end{array}$ & 1,57 \\
\hline $\begin{array}{c}7,3167-(1366,74) \\
\text { Xénon } 5 d[7 / 2]_{3}-6 p[5 / 2]_{2}\end{array}$ & $\begin{array}{l}2 \rightarrow 1 \\
1 \rightarrow 0 \\
0 \rightarrow-1\end{array}$ & $\begin{array}{l}1,730 \\
1,376 \\
1,022\end{array}$ & $\begin{array}{l}6 / 10 \\
3 / 10 \\
1 / 10\end{array}$ & $\begin{array}{l}2,42 \\
1,93 \\
1,43\end{array}$ & 2,20 \\
\hline $\begin{array}{c}7,7407 \\
\text { Néon } 4 p[5 / 2]_{2}-3 d[3 / 2]_{2}\end{array}$ & $\begin{aligned} 2 & \rightarrow \\
1 & \rightarrow \\
0 & \rightarrow-1 \\
-1 & \rightarrow-2\end{aligned}$ & $\begin{array}{l}1,600 \\
1,112 \\
1,356 \\
0,868\end{array}$ & $\begin{array}{l}2 / 10 \\
3 / 10 \\
3 / 10 \\
2 / 10\end{array}$ & $\begin{array}{l}2,24 \\
1,57 \\
1,89 \\
1,21\end{array}$ & $\begin{array}{l}\text { non mesuré } \\
\text { transition } \\
\text { inutilisable }\end{array}$ \\
\hline $\begin{array}{c}8,0088-(1248,631) \\
\text { Néon } 4 p^{\prime}[3 / 2]_{1}-3 d^{\prime}[5 / 2]_{2}\end{array}$ & $\begin{aligned} 1 & \rightarrow 0 \\
0 & \rightarrow-1 \\
-1 & \rightarrow-2\end{aligned}$ & $\begin{array}{l}0,685 \\
0,781 \\
0,877\end{array}$ & $\begin{array}{l}6 / 10 \\
3 / 10 \\
1 / 10\end{array}$ & $\begin{array}{l}0,96 \\
1,09 \\
1,25\end{array}$ & 1,0 \\
\hline $\begin{array}{c}7,4799-(1336,917) \\
\text { Néon } 4 p[3 / 2]_{2}-3 d[5 / 2]_{3}\end{array}$ & 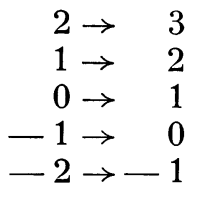 & $\begin{array}{l}1,027 \\
1,14 \\
1,249 \\
1,360 \\
1,47\end{array}$ & $\begin{array}{r}15 / 35 \\
10 / 35 \\
6 / 35 \\
3 / 35 \\
1 / 35\end{array}$ & $\begin{array}{l}1,44 \\
1,60 \\
1,75 \\
1,91 \\
2,06\end{array}$ & 1,40 \\
\hline $\begin{array}{c}7,6510-(1307,012) \\
\text { Néon } 4 p[5 / 2]_{2}-3 d[7 / 2]_{3}\end{array}$ & $\begin{array}{rr}2 \rightarrow & 3 \\
1 \rightarrow & 2 \\
0 & \rightarrow \\
-1 & \rightarrow \\
-2 & \rightarrow-1\end{array}$ & $\begin{array}{l}0,88 \\
0,95 \\
1,034 \\
1,112 \\
1,190\end{array}$ & $\begin{array}{r}15 / 35 \\
10 / 35 \\
6 / 35 \\
3 / 35 \\
1 / 35\end{array}$ & $\begin{array}{l}1,23 \\
1,33 \\
1,45 \\
1,57 \\
1,67\end{array}$ & 1,28 \\
\hline
\end{tabular}

où $g_{0 \imath}$ est le gain au centre de la raie $\sigma_{i}^{ \pm}$tel que $\sum_{i} g_{0 i}=g_{0}, g_{0}$ étant le gain au centre de la raie en l'absence de champ magnétique, $v_{i}^{\prime}=\frac{2\left(\nu-v_{0}\right)}{\Delta \nu_{\mathrm{D}}} \log 2$ et $\Delta v_{i}^{\prime}=\frac{2 \Delta v_{i}}{\Delta \nu_{\mathrm{D}}} \sqrt{\log 2}, \Delta \nu_{\mathrm{D}}$ étant la largeur Doppler.

Les gains $g_{0 i}$ sont obtenus à partir des formules classiques donnant l'intensité des composantes Zeeman [5].

Le tableau I indique les déplacements de fréquence $\Delta v_{i}(H)$ et les gains $g_{0 i}$ des composantes $\sigma_{i}^{ \pm}$des transitions laser étudiées. Les facteurs de Landé indiqués sont ceux figurant dans les tables de C. E. Moore [6].

I.2. VARIATION DE LA PUISSANCE LASER EN FONGTION DU CHAMP MAGNÉTIQUE. - La puissance délivrée par un laser à gaz multimodes (modes longitudinaux) dépend du nombre de modes $\nu_{n}$ contenus dans la courbe de gain, donc de la largeur de cette courbe, de l'écart $\Delta v_{n}=\frac{c}{2 L}$ entre deux modes et de la position du maximum de gain par rapport aux modes de résonance de la cavité. Du fait du champ magnétique, la fréquence qui correspond au maximum de gain n'est plus $\nu_{0}$ mais $\nu_{0}+\Delta v_{i}(H)$ pour une $e_{t}^{q}$ oscillation $\sigma_{i}^{+}$, ou $\nu_{0}-\Delta v_{i}(H)$ pour une oscillation $\sigma_{i}^{-}$. La position relative de ces fréquences et des modes $\nu_{n}$ de la cavité varie avec $\Delta v(H)$, c'est-à-dire avec le champ magnétique $H$. Il résulte de cette situation une variation de la puissance émise en fonction du champ magnétique.

Lorsque les pertes de la cavité résonnante sont faibles, on trouve (voir annexe) que la puissance est une fonction périodique continue du champ tant que le gain demeure constant. Par contre, la fréquence d'émission n'est pas une fonction continue du champ, l'oscillation laser n'ayant lieu qu'aux fréquences $\nu_{n}$ fixes si la longueur de la cavité est constante.

La courbe de variation de la puissance en fonction du champ $H$ est correctement représentée par la fonction elliptique $\theta_{3}(H, \chi)$, fonction périodique de $H$ et de minimums plus ou moins marqués selon la valeur 
du paramètre $\chi=\frac{\pi \delta^{2}}{\left(\Delta v_{\mathrm{n}}\right)^{2}}$ où $\delta=\frac{\Delta \nu_{\mathrm{D}}}{2 \sqrt{2 \log 2}}$, aux faibles valeurs de $\chi$ correspondant une importante modulation de la courbe de puissance.

La figure 1 montre la modulation de la puissance émise pour deux valeurs de $\frac{\Delta \nu_{D}}{\Delta v_{n}}$, les paramètres $P_{0}$ et $X$ étant identiques pour ces deux courbes, les fréquences $v_{n}$ des modes étant supposées fixes dans le temps.

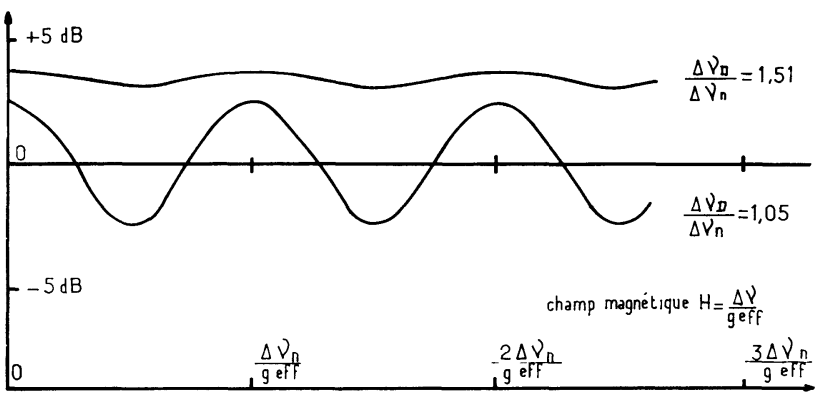

FIG. 1.

Expérimentalement, on constate que la courbe de puissance du laser à xénon est nettement plus modulée que celle du laser à néon, ce qui était prévisible puisque $\frac{\Delta v_{D} \text { (xénon) }}{\Delta v_{D} \text { (néon) }} \simeq 2$. Cependant, la courbe est moins modulée que ne le prévoit la formule théorique, cela étant sans doute dû aux déplacements de fréquence des modes liés aux variations de longueur de la cavité résonnante.

De la périodicité de la courbe de puissance, on déduit le déplacement de fréquence $\Delta \nu(H)$ et on calcule la valeur moyenne du facteur de Landé $g_{\text {eff }}$.

Le laser utilisé dans nos expériences a une cavité de $1,66 \mathrm{~m}$ de longueur, ce qui conduit à un écart $\Delta \nu_{n}$ de $90 \mathrm{Mc} / \mathrm{s}$ entre modes longitudinaux.

La largeur Doppler d'une transition du néon est de $120 \mathrm{Mc} / \mathrm{s}$ à $8 \mu$, celle du xénon de $60 \mathrm{Mc} / \mathrm{s}$ à $7,3 \mu$. (Il est possible que la largeur naturelle $\delta v_{N}$ des raies du xénon soit aussi de l'ordre de $60 \mathrm{Mc} / \mathrm{s}$, ce qui expliquerait la modulation moins importante de la courbe de puissance.)

En plus du phénomène de modulation décrit précédemment, on observe une diminution de la puissance laser lorsque le champ magnétique croît. Cet effet est lié à la modification du processus de collision entre atomes et conduit à la destruction de l'inversion de population. Ce phénomène est peu marqué pour les transitions de fort gain $(3,39 \mu$ et $7,70 \mu)$, mais amène rapidement l'extinction de l'oscillation laser des transitions faibles. En général, l'extinction est presque complète lorsque le champ atteint 1600 œrsteds, ce qui correspond à un déplacement de fréquence de $\pm 0,1 \mathrm{~cm}^{-1}$ et à une variation relative de puissance de 1 à $\frac{1}{30}$.

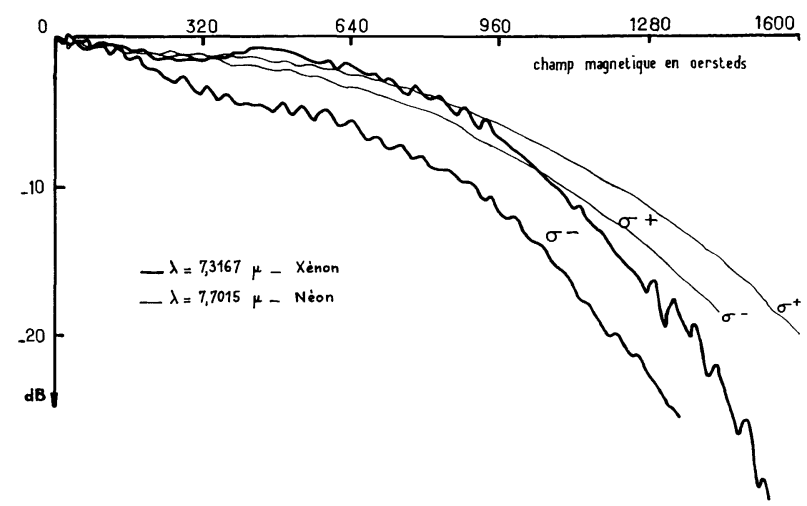

FIG. 2. - Variation de la puissance en fonction du champ magnétique.

La figure 2 montre la variation de puissance des raies à $7,7015 \mu$ du néon et de la raie à $7,3167 \mu \mathrm{du}$ xénon. La cavité résonnante est composée d'un miroir à couche d'or de $1 \%$ de transmission $(R \simeq 97 \%)$ et d'un miroir à réflexion maximale $(R \simeq 98 \%)$.

I.3. Garagtéristiques SPegtrales De L'Émission LASER. - Aux faibles valeurs du champ magnétique $(H<500 \mathrm{E})$, les composantes $\sigma_{i}^{ \pm}$ont des fréquences qui diffèrent d'une quantité inférieure à la largeur Doppler.

Aux valeurs élevées du champ magnétique $(H>500 \mathbb{E})$, le gain $g_{0 i}$ des composantes $\sigma^{ \pm}$est réduit par suite des effets de plasma et, seule la composante $\sigma_{i}^{ \pm}$ de plus grand gain peut osciller, car c'est alors la seule qui conserve un gain supérieur aux pertes. De plus, le phénomène de compétition entre raies fait que la composante $\sigma_{i}^{+}$de plus grand gain élimine la composante $\sigma_{i}^{-}$de faible gain issue du même niveau et vice versa.

Des observations précédentes, on conclut que le déplacement de la fréquence d'émission laser doit être proche du déplacement $\Delta \nu(H)$ de la composante $\sigma_{\mathrm{i}}$ de plus grand gain. Ceci est assez bien vérifié par l'expérience (voir tableau I).

Par ailleurs, l'émission laser a lieu aux fréquences des modes contenus dans la courbe de gain. Or, la fréquence $v_{n}$ d'un mode fluctue rapidement autour d'une valeur $v_{n 0}$ fixe pendant le temps de mesure (2 $\mathrm{mn})$, les fluctuations de fréquence traduisant les variations de longueur de la cavité résonnante. Il s'ensuit que la fréquence de l'oscillation laser se déplace à l'intérieur de la raie Doppler. La distribution spectrale du rayonnement laser est donc représentée par une courbe gaussienne, centrée en $\nu_{0} \pm \Delta \nu(H)$, de largeur $\delta v_{\mathrm{L}}$ inférieure ou égale à la largeur Doppler $\Delta \nu_{D}$

La largeur spectrale $\delta v_{\mathrm{L}}$ dépend du gain de la transition et des pertes, donc du coefficient de transmission du miroir de sortie. Pour une transition de gain moyen (quelques décibels) et un miroir de faible transmission $(T \leqslant 1 \%)$, on observe $\delta v_{\mathrm{L}} \simeq \Delta v_{\mathrm{D}}$, soit $\delta v_{\mathrm{L}} \simeq 4.10^{-3} \mathrm{~cm}^{-1}$ pour le néon. 
I.4. INTÉRÊT DU LASER A FRÉQUENGE AGGORDÉE EN SPEGTROSGOPIE. - On montre facilement que, lorsqu'on analyse une raie d'absorption de profil $A(\nu)$ avec une source de profil $A_{\mathrm{L}}(v)$, la courbe d'absorption résultante $A^{\prime}(\nu)$ est donnée par :

$$
A^{\prime}(\nu)=A(\nu) * A_{\mathrm{L}}(\nu)
$$

(* produit de convolution).

Si $A(\nu)$ et $A_{\mathrm{L}}(\nu)$ sont des raies gaussiennes, de largeurs respectives $\delta$ et $\delta v_{\mathrm{L}}, A^{\prime}(\nu)$ est une raie gaussienne de largeur $\left[\delta^{2}+\left(\delta v_{\mathrm{L}}\right)^{2}\right]^{1 / 2}$. De même, si $A(v)$ et $A_{\mathrm{L}}(\nu)$ sont des raies lorentziennes, $A^{\prime}(\nu)$ est lorentzienne de largeur $\delta+\delta v_{\mathrm{L}} \quad$ [10].

On dispose, avec le laser à fréquence accordée par effet Zeeman, d'une source infrarouge de fréquence variable sur une plage de $\pm 0,1 \mathrm{~cm}^{-1}$ et de largeur spectrale de quelque $10^{-3} \mathrm{~cm}^{-1}$. Cela permet de réaliser un spectromètre infrarouge de très haute résolution pouvant résoudre des raies d'absorption séparées de moins de $0,005 \mathrm{~cm}^{-1}$.

Expérimentalement, on a résolu deux raies de la branche $Q$ du méthane, distantes de $0,003 \mathrm{~cm}^{-1}$ (voir fig. 5). La fréquence laser utilisée est $\nu_{0}=1298,443 \mathrm{~cm}^{-1}$ (transition $\lambda=7,7015 \mu \mathrm{du}$ néon), la cavité résonnante étant composée d'un miroir à couche d'or à réflexion maximale, $R \simeq 98 \%$, et d'un miroir à couches multidiélectriques, $T \simeq 2 \%, R \simeq 95 \%$.

II. Dispositif expérimental. - Le laser est du type à miroirs internes. Cette géométrie a été adoptée pour conserver les polarisations circulaires liées à l'effet Zeeman et on évite ainsi l'emploi de fenêtres qui occasionnent des pertes. La décharge, de 1,28 $\mathrm{m}$ de longueur, est excitée en continu, la tension est de l'ordre de $5000 \mathrm{~V}$ et le courant peut atteindre $250 \mathrm{~mA}$. Le tube comporte une anode en acier inoxydable et une cathode chaude à oxydes.

La cavité Fabry-Perot a 1,66 m de longueur et est composée de miroirs de $2 \mathrm{~m}$ de rayon de courbure. L'un des miroirs est à réflexion maximale (couche d'or épaisse, $R \simeq 98 \%$ ), l'autre a, selon les besoins, de 0,5 à $2 \%$ de transmission (couche d'or mince ou couches multidiélectriques).

Pour éviter les vibrations mécaniques qui créent des fluctuations de fréquence et de puissance, on a monté le laser sur un banc en granit posé sur des amortisseurs vibrachocs.

Presque toutes les transitions laser connues [11], [12] entre 4 et $9 \mu$ ont été observées.

La transformation en polarisation rectiligne des polarisations circulaires des transitions $\sigma^{+}$et $\sigma^{-}$est obtenue à l'aide d'un prisme de Fresnel en $\mathrm{NaCl}$ agissant comme une lame quart d'onde.

Le polariseur qui permet de choisir la composante $\sigma^{+}$ (fréquence $\nu_{0}+\Delta v$ ) ou $\sigma^{-}$(fréquence $\nu_{0}-\Delta v$ ) est du type à transmission et comporte trois lames minces de sélénium (épaisseur $3 \mu$ ) obtenues par évaporation sous vide sur support en collodion [13]. Le taux de polarisation obtenu dépasse $99 \%$.

Le champ magnétique est produit par des bobines (six bobines de $20 \mathrm{~cm}$ comportant vingt couches de 900 spires) et atteint $1800 œ$ pour un courant de $17 \mathrm{~A}$.

Un système à double faisceau a été réalisé pour s'affranchir de la variation de puissance en fonction du champ, des fluctuations aléatoires et de l'absorption atmosphérique.

Avant de pénétrer dans la cuve à réflexions multiples (quatre passages de $25 \mathrm{~cm}$ ), le faisceau est

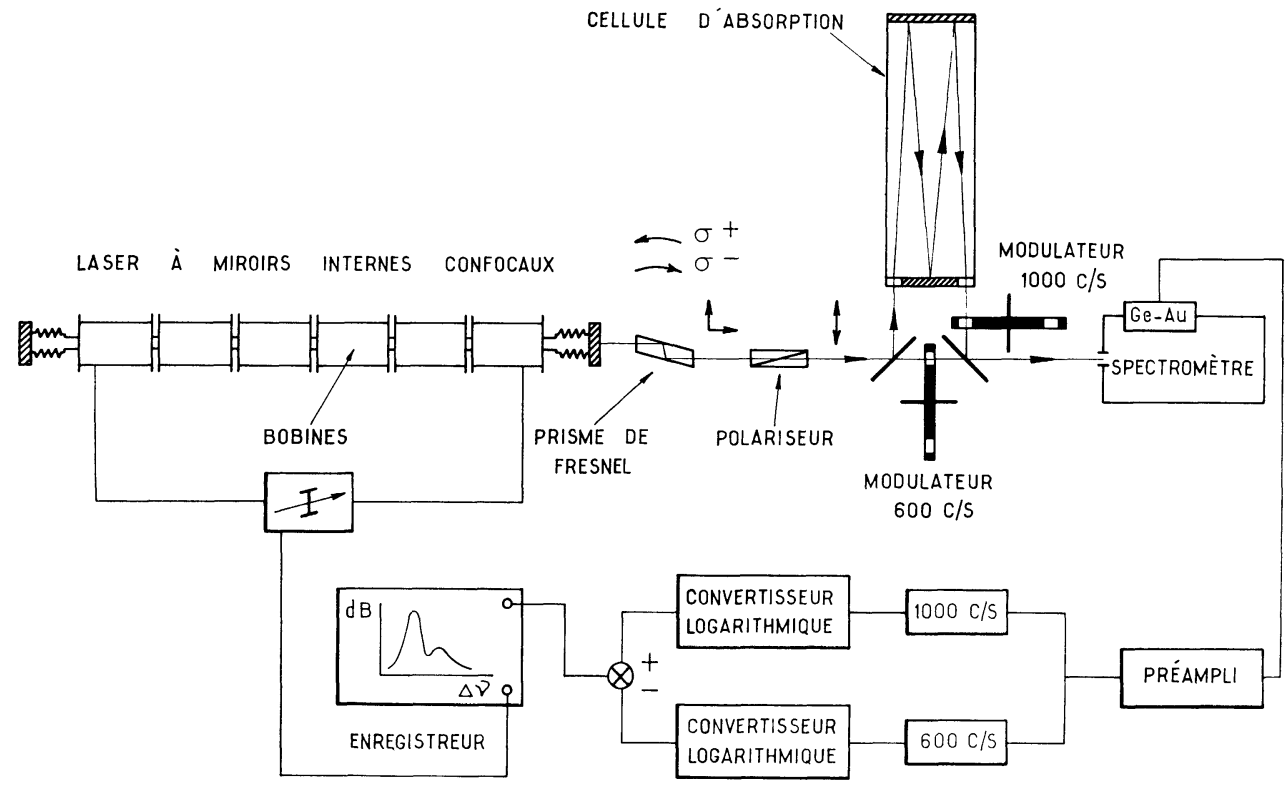

FIG. 3. - Principe du spectromètre utilisant l'effet Zeeman. 
divisé, à l'aide de deux lames séparatrices à $45^{\circ}$, en deux parties; l'une traverse la cuve et l'autre est utilisée comme faisceau de référence. Le premier faisceau d'intensité $I$ est modulé à $1000 \mathrm{c} / \mathrm{s}$, le second d'intensité $I_{0}$ à $600 \mathrm{c} / \mathrm{s}$. Un spectromètre Perkin Elmer, équipé d'une cellule Ge-Au et d'un réseau à $75 \mathrm{t} / \mathrm{mn}$, permet de choisir la transition laser pour laquelle on désire mesurer l'absorption du gaz contenu dans la cuve. Une lentille en KRS 5 focalise le faisceau sur la fente d'entrée du spectromètre.

Le signal fourni par la cellule Ge-Au est amplifié par un préamplificateur P.A.R. et par deux amplificateurs accordés à $1000 \mathrm{c} / \mathrm{s}$ et $600 \mathrm{c} / \mathrm{s}$. Ces deux signaux sont ensuite convertis (convertisseurs Moseley) en deux signaux logarithmiques que l'on soustrait pour obtenir le rapport $I / I_{0}$ en décibels. La réjection mutuelle des deux voies est de $35 \mathrm{~dB}$.

Le signal $I / I_{0}$ est appliqué à la voie Y d'un enregistreur XY Moseley dont la voie $\mathrm{X}$ reçoit un signal proportionnel au champ magnétique. Ce dispositif ( fig. 3) permet de tracer directement la courbe d'absorption en fonction du champ, donc de la fréquence.

III. Résultats obtenus en spectroscopie. - Nous avons étudié quelques bandes d'absorption du méthane, de l'éthane, du propane et de l'hexafluorure de soufre. La bande d'absorption de la vibration $\nu_{4}$ du méthane a été analysée à l'aide des raies laser du néon à $7,4799 \mu, 7,7015 \mu$ et $7,7655 \mu$ et la bande de la vibration $\nu_{6}$ de l'éthane à l'aide de la raie à $7,3167 \mu$ du xénon.

Des raies d'absorption fines ont été observées dans
$\mathrm{CH}_{4}$ et $\mathrm{C}_{2} \mathrm{H}_{6}$ avec une cuve d'absorption de $1 \mathrm{~m}$ et des pressions de quelques torr.

Nous avons mis en évidence la structure rotationnelle compliquée d'une bande d'absorption du propane (vibrations $\nu_{6}, \nu_{18}$ ou $\nu_{22}$ ) et de la bande $\nu_{2}+\nu_{4}$ de l'hexafluorure de soufre. La structure rotationnelle n'est pas résolue par suite du faible espacement des niveaux de rotation dû au poids élevé de la molécule $\left(\mathrm{SF}_{6}\right)$ et aux interactions de Coriolis qui décomposent en sous-niveaux chaque niveau de rotation $\left(\mathrm{SF}_{6}\right.$, $\mathrm{C}_{3} \mathrm{H}_{8}$ ).

Les résultats sont présentés dans le tableau II. On a indiqué la fréquence de la raie laser, les fréquences des raies d'absorption observées à l'aide de cette raie et les intensités d'absorption.

Les fréquences des raies $\mathrm{R}_{5}\left(\mathrm{~F}_{1}^{\prime}\right)$ et $\mathrm{P}_{3}\left(\mathrm{~F}_{2}\right)$ de $\mathrm{CH}_{4}$ (fig. 4) sont légèrement différentes de celles précé-

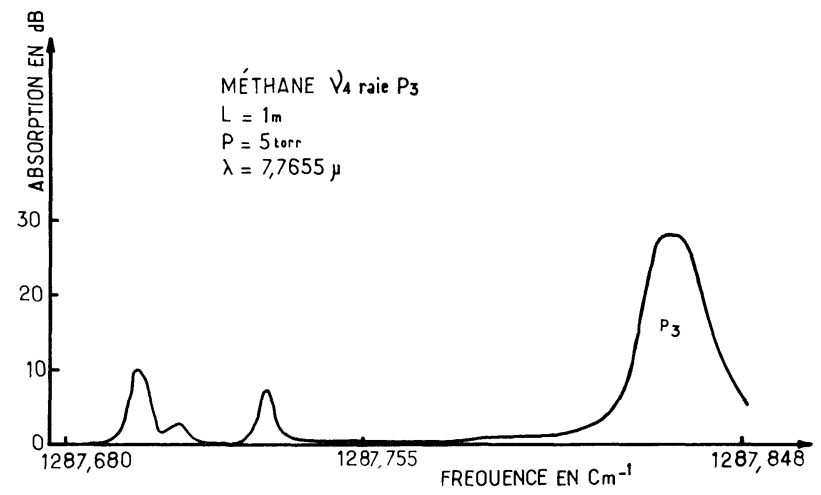

FIG. 4.

\section{TABLEAU II}

Fréquences laser galqulées a partir des tables de C. E. Moore [6]

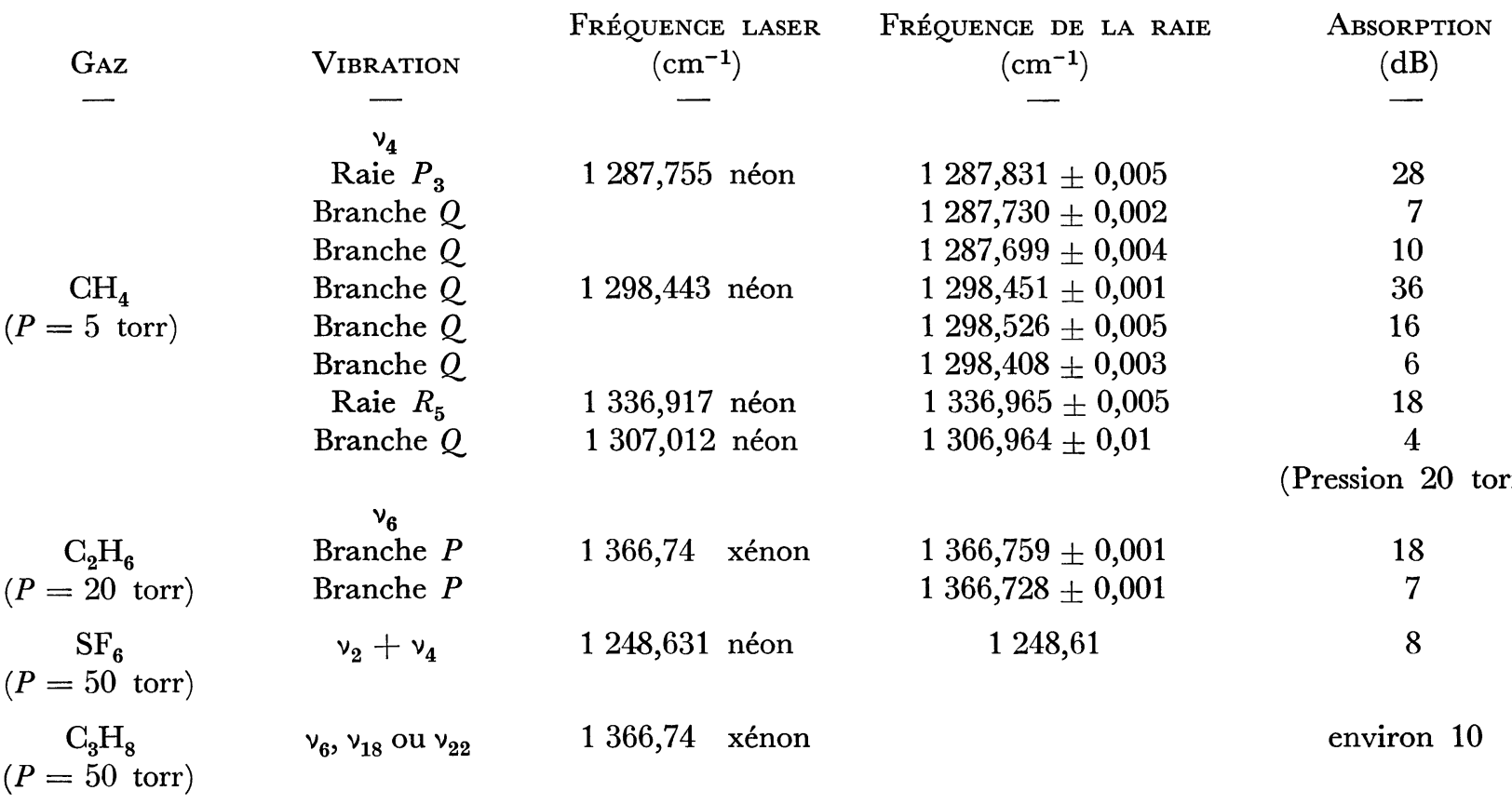


demment indiquées par les spectroscopistes, mais sont en très bon accord avec celles calculées par J. MoretBailly [14].

Les figures 4,5 et 6 montrent les raies observées dans $\mathrm{CH}_{4}$ et $\mathrm{SF}_{6}$.

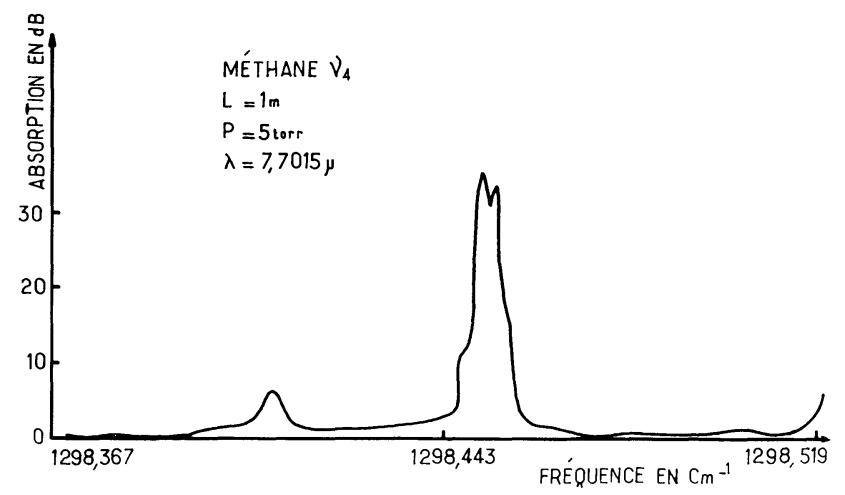

FIG. 5.

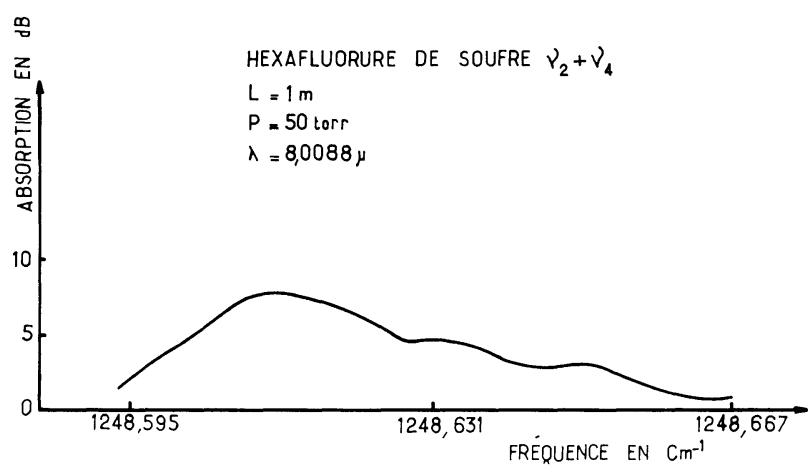

FIG. 6.

Conclusion. - Gette étude a montré l'intérêt du système de spectroscopie à très haute résolution utilisant un laser à fréquence accordée par effet Zeeman.

Ce nouveau dispositif permet d'analyser, avec une résolution meilleure que $0,005 \mathrm{~cm}^{-1}$, le spectre de rotation-vibration des molécules dont les fréquences d'absorption coïncident avec celles des oscillations laser que l'on sait produire.

On a pu séparer des raies extrêmement serrées de la branche $Q$ de la vibration $\nu_{4}$ du méthane et on a montré que les spectres d'absorption de certaines molécules présentaient une structure rotationnelle extrêmement compliquée.

\section{ANNEXE}

Calcul de la puissance en fonction du champ magnétique. - La puissance d'un laser à gaz multimodes est donnée par [7] :

$$
P=P_{0} X^{2} \sum_{n}\left(\mathrm{e}^{-\left(\frac{v_{n}-v_{0}}{\delta}\right)^{2}}-\frac{1}{X^{2}}\right) \quad \text { si } \quad P \geqslant 0
$$

où $\nu_{n}$ est la fréquence des modes longitudinaux, $\nu_{0}$ la fréquence du centre de la courbe de gain et

$$
\frac{\delta}{\sqrt{2}}=\Delta \nu_{\mathrm{c}}=\frac{\Delta \nu_{\mathrm{D}}}{2 \sqrt{\log 2}}
$$

où $\Delta \nu_{D}$ est la largeur Doppler.

$$
X=\frac{g_{0} L}{a+t} \text { est le rapport du gain non saturé au }
$$

gain saturé, $g_{0}$ le gain au centre de la raie, $L$ la longueur du milieu amplificateur, $t$ le coefficient de transmission des miroirs et $a$ représente les pertes par diffusion, diffraction, etc.

Nous ne considérerons que le cas où les pertes totales sont suffisamment faibles par rapport au gain pour qu'il y ait toujours au moins un mode à l'intérieur de la courbe de gain, quelle que soit la position de $v_{0}$ par rapport aux $v_{n}$.

Cette condition nécessite que l'on ait

$$
2 \delta \sqrt{2 \log } X>\Delta v_{n}
$$

et alors la puissance est une fonction continue du champ magnétique représentée par :

$$
P(v)=P_{0} X^{2}\left(\sum_{n=M}^{N} \mathrm{e}^{-\left(\frac{n \Delta v_{n}-v}{\delta}\right)^{2}}-\frac{1}{X^{2}}\right)
$$

s'il y a $M+N+1$ modes dans la courbe de gain. $\nu$ est la fréquence du centre de la courbe de gain.

Lorsque les inégalités $\delta \sqrt{2 \log X}>\frac{\Delta \nu_{n}}{z}$ et $X \gg 1$ sont satisfaites, on peut étendre la sommation sur $n$ de $-\infty$ à $+\infty$ (erreur de l'ordre de $1 / X^{2}$ ) et on obtient :

$$
P(\nu) \simeq P_{0} X^{2}\left(\sum_{n=-\infty}^{+\infty} \mathrm{e}^{-\left(\frac{n \Delta v_{n}-v}{\delta}\right)^{2}}\right) .
$$

En appliquant la formule sommatoire de Poisson [8] à la fonction $\mathrm{e}^{-\left(\frac{X-v}{\delta}\right)^{2}}$ et à sa transformée de Fourier $\delta \sqrt{ } \bar{\pi} \mathrm{e}^{2 i \pi v} y \mathrm{e}^{-\pi^{2} y^{2} \delta^{2}}$, on obtient :

$$
\sum_{n=-\infty}^{+\infty} \mathrm{e}^{-\left(\frac{n \Delta v_{n}-v}{\delta}\right)^{2}}=\frac{\delta \sqrt{\pi}}{\Delta \nu_{n}} \sum_{-\infty}^{+\infty} \mathrm{e}^{\frac{2 i \pi v_{n}}{\Delta v_{n}}} \mathrm{e}^{-\pi^{2} n^{2}\left(\frac{\delta}{\Delta v_{n}}\right)^{2}} .
$$

On reconnaît à droite la fonction elliptique Theta :

$$
\theta_{\mathbf{3}}(v, \chi)=\theta_{3}\left(\frac{\nu}{\Delta \nu_{n}}, \pi\left(\frac{\delta}{\Delta \nu_{n}}\right)^{2}\right)
$$

d'où l'expression de la puissance laser :

$$
P(\nu)=\frac{\delta \sqrt{ }{ }^{\prime} \pi}{\Delta \nu_{n}} \theta_{3}\left(\frac{\nu}{\Delta \nu_{n}}, \pi\left(\frac{\delta}{\Delta \nu_{n}}\right)^{2}\right) .
$$

La fonction $\theta_{3}(v, \chi)$ a été tabulée par JahnkeEmde-Lösch [9]. Les tables donnent, d'une part $\theta_{3}(v, \alpha)$, et d'autre part $\alpha$ en fonction de $\log q=$ $0,434 \pi \chi$. La figure 1 montre l'aspect de la courbe $P(\nu)$ pour deux valeurs de $\frac{\delta}{\Delta \nu_{n}}, X$ et $P_{0}$ étant fixes.

Manuscrit reçu le 17 mars 1967. 


\title{
BIBLIOGRAPHIE
}

[1] Gerritsen (H. J.), Helier (M. E.), " High Resolution tuned Laser Spectroscope ", Appl. Optics, Suppl. 2 on Chemical Lasers, p. 73-80, 1965.

GERRITSEN (H. J.), "Tuned-Laser Spectroscopy of Organic Vapors ", Physics of Quantum Electronics, New York, Mac Graw Hill, 1966, 581.

[2] Sakurai (K.), Shimoda (K.), " Tunable Infrared Maser Spectrometers ", Japan J. Appl. Phys., 1966, 5, 10, 938-947.

[3] BRUneT (H.), " High Resolution Spectroscopy by Zeeman-tuned Infrared Laser ", I.E.E.E. J. of Quantum Electronics, QE 2, 9, 382-384, septembre 1966.

[4] FORK (R. L.), PATEL (C. K. N.), " Broadband Magnetic Field Tuning of Optical Masers ", $A p p l$. Phys. Letters, May 1963, 2, 180-181.

[5] WhITE (H. E.), "Introduction to Atomic Spectra ", New York, Mac Graw Hill, 1964, p. 221.

[6] Moore (C. E.), "Atomic Energy Levels ", N.B.S., Washington D.C., Circular 467, août 1952.
[7] Rigrod (W. W.), "Gain Saturation and Output Power of Optical Masers ", J. Appl. Phys., septembre 1963, 34, 9, 2602-2609.

[8] SchwarTZ (L.), " Méthodes mathématiques pour les Sciences Physiques ", Paris, Hermann, 1961, p. 217-218.

[9] JAHNKE, EMDE, LöSCH, "Tables of Higher Functions ", New York, Mac Graw Hill, 1960, p. 82-94.

[10] SchwarTz (L.), loc. cit., p. 127-128.

[11] Patel (C. J. N.), "Noble Gas Optical Maser Lines at Wavelengths between $2 \mu$ and $35 \mu$ ", Phys. Rev., mars 1964, 133, $6 \mathrm{~A}, 1476-1486$.

[12] Brochard (J.), LIBERman (S.), "Émission stimulée de nouvelles transitions infrarouges de l'hélium et du néon ", C. R. Acad. Sc. Paris, juin 1965, 260, 6827-6829.

[13] LENFANT (R.), "Un polariseur dans l'infrarouge ", La recherche aéronautique, juillet-août 1961, 83, 55-57.

[14] Moret-Bailly (J.), Thèse, Paris, 10 octobre 1961, p. 73-74.

\section{APPAREILlAGES DE MESURE DE LA PIÉZORÉSISTANGE DES SEMICONDUGTEURS}

\author{
Par G. WEILL, \\ Laboratoire de Magnétisme et de Physique du Solide, G.N.R.S. (92), Meudon-Bellevue.
}

\begin{abstract}
Résumé. - L'amplification sélective de signaux alternatifs permet d'atteindre la grande sensibilité exigée par les mesures de piézorésistance. Ce résultat est obtenu soit par l'application à l'éprouvette d'une contrainte alternative, soit par l'utilisation du courant alternatif dans le circuit de mesure. Les deux appareillages décrits sont utilisables dans des domaines complémentaires. Le second, en outre, peut trouver d'utiles applications à des mesures précises de résistivité, d'effet Hall ou de magnétorésistance.
\end{abstract}

Abstract. - Measurement of piezoresistance coefficients in semiconductors requires a high sensitivity which can be achieved through selective amplification of alternating signals. Such a result has been obtained either by applying an alternating stress on the sample or by working with alternating currents in the circuits. Both techniques are described, each of which being used in the case when the other fails to give satisfactory performance. Furthermore, the lat ter may be used when precision measurements of resistivity, Hall effect or magnetoresistance are required.

I. Introduction. - Depuis les travaux de C. S. Smith [1] sur l'effet de piézorésistance dans le germanium et le silicium, l'étude de ce phénomène s'est avérée capable de fournir des indications utiles sur la structure de bandes des semiconducteurs. Rappelons qu'il s'agit de mesurer la variation des propriétés électroniques du matériau (résistivité électrique et éventuellement concentration des porteurs de charge), sous l'effet d'une contrainte mécanique.
Des mesures sous faible contrainte permettent de déterminer, d'une part les caractères de symétrie de la structure de bandes, et d'autre part des paramètres fondamentaux tels que les coefficients de pression des énergies de bord de bande et des masses effectives, et, dans le cas particulier où les bandes se déplacent linéairement avec la pression sans se déformer, les coefficients du potentiel de déformation.

G'est ainsi par exemple que Smith [1] a pu confir- 